\title{
Use of the wound healing trajectory as an outcome determinant for acute wound healing
}

\author{
MICHAEL G. FRANZ, MDa; M. ANN KUHN, MDb; TERRY E. WRIGHT, MDb; THOMAS L. WACHTEL, MDb; MARTIN \\ C. ROBSON, MD
}

\begin{abstract}
Accurate and clinically practical methods for measuring the progress of acute wound healing is necessary before interventions designed to optimize and even accelerate acute wound healing can be applied. Complete wound closure rates and operative wound closure severity are irrelevant to most acute wounds since most are closed at the time of primary tissue repair and remain closed throughout healing. Analogous to chronic wound closure, the rate of increase of incision tensile strength progressively decreases as time passes and $100 \%$ unwounded tissue strength is never achieved making the endpoint definition of "healed" vague. Conceptualizing acute wound healing in terms of its design elements with reintegration into a final outcome lends itself to the description of acute wound healing as a mathematical trajectory. Frequently such an equation is a rate expressing the change in an acute healing parameter, most often tensile strength, over time. Such an approach also normalizes misinterpretations in analysis or errors in theory developed by measuring healing parameters at fixed points in time. Distributions of fractional strength gain times (e.g., 85\% normal strength) can be determined using statistical methodology similar that used for failure time of survival analysis. Preclinical studies show that acute wound healing trajectories can be shifted to the left from a "normal" or "impaired" curve to an accelerated or more "ideal" curve. A useful method for measuring acute wound healing outcomes is therefore required before the basic science of acute wound healing is inevitably applied to the problem of acute surgical wounds. (WOUND REP REG 2000;8:511-516)
\end{abstract}

Unlike chronic wounds, most acute wounds heal. The problem with acute wounds is that they take a long time to heal, are meanwhile disabling, and most often result in scar. The magnitude of the problem is emphasized by the nearly 50 million surgical procedures performed each year in the United States alone. ${ }^{1}$ Recovery from these procedures conservatively required 250 million patientdays in lost productivity and billions of dollars in lost or supplemented earnings. Surgical wound complications obviously add to these numbers. Despite technical ad-

From the Department of Surgerya, University of Michigan, Ann Arbor, Michigan and the Institute for Tissue Regeneration, Repair and Rehabilitation', Bay Pines Veterans Affairs Medical Center, Bay Pines, Florida.

Reprint requests: Michael G. Franz, MD, Ann Arbor VAMC, Surgery Service (1 12), 2215 Fuller Road, Ann Arbor, MI 48105-9915. Fax: (727) 319-1038; Email: mfranz@ com 1.med.usf.edu.

Copyright (C) 2000 by The Wound Healing Society.

ISSN: 1067-1927\$15.00+0

\section{$\mathrm{P}_{\mathrm{T}} \mathrm{O}_{2} \quad$ Oxygen partial pressure}

vances, the incidence of fascial dehiscence, incisional hernia formation, gastrointestinal anastamotic breakdown, peritoneal adhesion formation, anastamotic stricture, hypertrophic scar, and keloid, has not declined substantially over the past 50 years of modern surgery. ${ }^{2}$ In 1997 the National Center for Health Statistics reported more than 100,000 operations annually for recurrent inguinal hernia, 110,000 incisional (ventral) hernia repairs and an approximately $10-30 \%$ leak rate following pancreatic and colorectal operations. ${ }^{1}$ These are just a few examples of acute wound healing failure.

Our increasingly sophisticated understanding of the basic cellular and molecular mechanisms of acute tissue repair has yet to be translated into meaningful clinical application. As a starting point, clinical scientists are borrowing from the vast experience of those who study 
and treat chronic wounds and attempting to adapt this to the problem of acute wounds. Unfortunately, no universally acceptable method for measuring or describing chronic wound healing exists. ${ }^{3,4}$ Complete wound closure rates and operative wound closure severity are irrelevant to most acute wounds since most are closed at the time of primary tissue repair and remain closed throughout healing. In addition, there are inherent ethical and practical difficulties in manipulating otherwise "normal" human acute wounds. Therefore, as acute wound therapy enters the clinical realm, newer concepts will be needed to describe the outcomes of acute wound healing.

The morbidity associated with acute wounding is comprised of two main components: the amount of tissue injury and the duration of tissue injury. The revolution that occurred with the development of minimally invasive surgical procedures proved that reducing the amount of surgical incision dramatically shortens recovery times. ${ }^{5}$ Unfortunately, minimal access is not always possible and large incisions and excisions remain an integral part of most surgical disciplines. Clearly the amount of acute wounding will remain an important component of any measure of acute wound healing success. The duration of the acute wound burden is a result of the long period of time required for tissue regeneration or repair to occur after injury. Weeks and sometimes months are necessary before the injured tissue is returned to its normal structure and function, regardless of which tissue or organ type is considered.$^{6,7}$ Normal tissue strength is never achieved in structures such as the gastrointestinal tract, vascular system or abdominal wall where the recovery of tissue tensile strength over time is a meaningful measure of acute healing.

Modern wound healing research has begun to elegantly describe the complex but well orchestrated series of cellular and molecular events that must sequentially occur in order to achieve successful tissue repair following injury. ${ }^{8}$ From a bioengineering perspective, these time-dependent design elements of the acute healing process integrate into the expected outcome that is usually a scar. The classic design elements of acute wound healing include the immediate processes of hemostasis and platelet degranulation, the local and then systemic host inflammatory response, and the production and release of potent peptide messengers responsible for initiating and propagating the influx of humoral and cellular mediators of the fibroproliferative response into the wound. ${ }^{9}$

The early acute wounding response is at the same time pleiotropic. Many of the immediate inflammatory response signals also activate early tissue repair. ${ }^{10,11}$ The arrival of fibroblasts into the provisional matrix of the acute wound and certainly the synthesis of collagen and the permanent extracellular matrix most often follow a predictable temporal pattern. Deterrents to acute healing increase the amount of time required for shifting the acute wound from a predominantly inflammatory state to a predominantly repair state. These include the amount of wound contamination and infection, tissue perfusion, tissue type, presence of foreign bodies, and host comorbidities. ${ }^{12}$ Accurately measuring the progression of acute wound healing is therefore further complicated by the many variables that affect it. Acute tissue repair does not occur in a vacuum and many internal and external factors can influence the progress of acute wound healing. The time-dependent integration of each of these subinfluences on the already complex process of tissue repair ultimately define the final wound healing trajectory.

Conceptualizing the complex process of acute wound healing in terms of its design elements with reintegration into a final outcome lends itself to the description of acute wound healing as a mathematical trajectory that can be measured. Most often this equation will be in the form of a rate, or change in an acute healing parameter over time. Such an approach also minimizes misinterpretations in analysis or errors in theory developed while considering acute wound healing as a static phenomenon and measuring healing parameters at fixed points in time. The classic acute wound trajectories for macrophage response, collagen production, and wound breaking strength illustrate the concept well. Isolated observations on day two compared to day 60 might lead to the conclusion that the early macrophage response is bad for acute healing since it is temporally associated with a delay in both collagen production and gain in wound breaking strength (Figure 1). Later, as the wound macrophage number diminishes, collagen production is accelerated and wound breaking strength rises. Experimental and clinical experience has proven, however, that macrophages are required for an acute wound to heal. ${ }^{13}$ Clinical and experimental data have also shown that a persistently elevated wound macrophage population that fails to diminish is associated with a delay in wound healing as manifested by a prolonged inflammatory response. ${ }^{14}$ It is only through an understanding of the time-dependent relationship between acute wound macrophage number, collagen production, and ultimately wound breaking strength that the mechanism of acute wound healing becomes clearer. For example, perhaps it is the ratio of acute wound collagen content to macrophage number over time that more accurately describes the optimum healing pathwaythan either element considered alone (Figure 2).

The cumulative goal of most wound healing research 


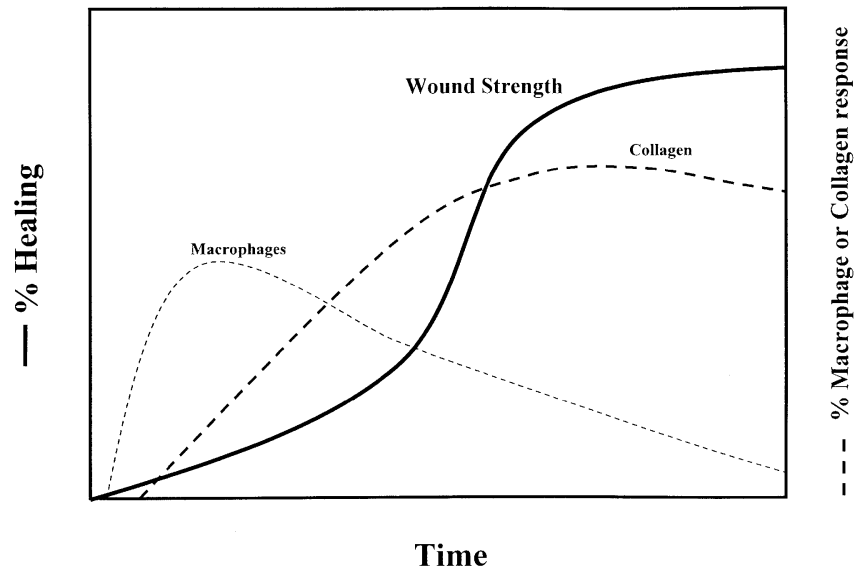

Figure 1. Acute wound healing is a dynamic process. Each of the cellular and molecular components of the acute wound healing cascade, as illustrated by the classic macrophage and collagen responses, integrate into the resultant wound strength trajectory. A comprehensive measure of the progress of acute wound healing should therefore account for these interactions and normalize for them over time.

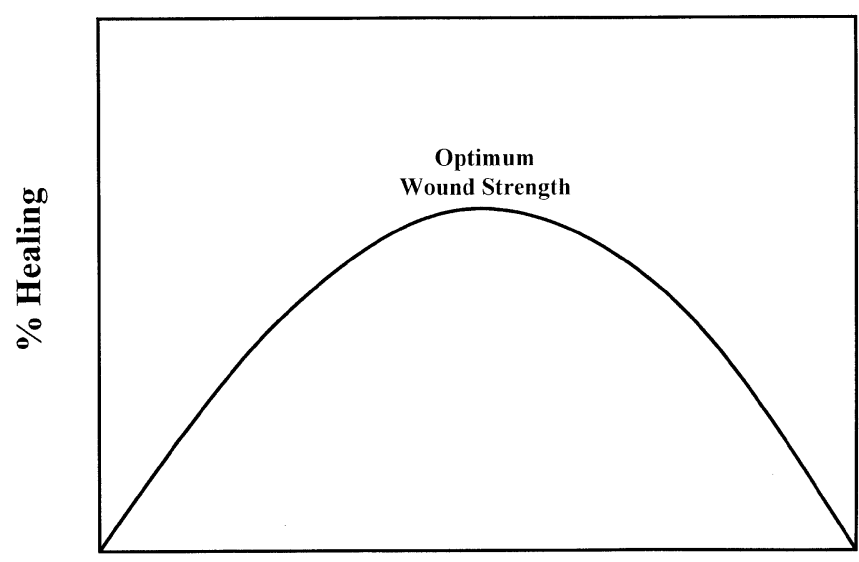

Ratio Collagen to Macrophage Number

Figure 2. A comparison of the many acute wound healing trajectories may also identify important interrelationships between the individual design elements of acute tissue repair. The ratio of acute wound collagen content to macrophage number, for example, may be more important to determining the progress of acute wound healing than either factor considered alone.

is to discover products and processes that can accelerate wound healing in humans..$^{15}$ Our laboratory is attempting to develop strategies for reducing the amount of time required for acute tissue repair following injury. At a meeting sponsored by the NIH and FDA in 1993 entitled, "Clinical Trial Issues in Topical Wound Healing Biologics," endpoints or outcome measures were discussed. Wound closure rates, operative wound closure severity, and quality of life endpoints were suggested, in addition to using the Wound Healing Society's definitions of "ideal" or "acceptable" wound healing and complete closure. ${ }^{16,17}$ To date most efforts to shorten the time of healing have been directed to chronic wounds. Many clinical trials involving the application of new drugs, biologics, and devices have been conducted during the past 10 years in an attempt to accelerate healing of chronic wounds with mixed success.

It is our belief that by accelerating acute wound healing trajectories it will be possible to both reduce the amount of incisional disability associated with normal acute healing and reduce the number of surgical complications resulting from acute wound failure. The problem is it is difficult to measure outcomes of acute incisional healing. Clinically evident incisional dehiscences occur in less than $3 \%$ of operative cases. ${ }^{2}$ Incisional hernias, however, still occur in over 200,000 cases per year, but this is less than $5 \%$ of all abdominal operations. ${ }^{1}$ We suggest using wound healing trajectories (e.g., gain in incisional healing strength vs. time) as outcome measures for acute wound healing. Wright et al. recently suggested that "normal" and "impaired" healing exist as a continuum and are a compromise from "ideal" healing (Figure 3 ). ${ }^{18}$ In attempting to accelerate acute incisional healing depicted by a breaking strength or tensile strength vs. time curve, they discuss the concept of moving the wound healing process in the direction of the "ideal". This essentially shifts the breaking strength vs. time curve to the left. Certainly, it is well known that diseases such as diabetes mellitus and chronic renal fail-

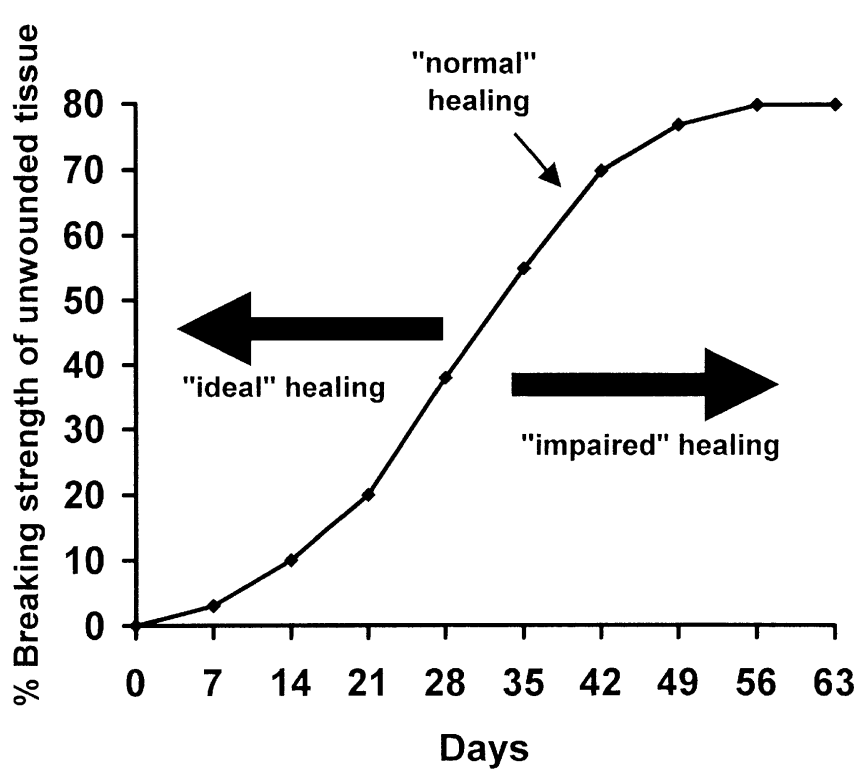

Figure 3. Ideal, normal and impaired acute wound healing may exist as a continuum. The pattern of a particular acute wound healing trajectory may therefore be conceptualized as a "shift to the left" (accelerated) or a "shift to the right" (delayed). 
ure as well as medications such as steroids impair healing and shift the acute wound healing trajectory to the right. ${ }^{19,20}$

Because healing of open wounds follows an exponential curve with the rate of change of wound area progressively decreasing as the residual wound area approaches total closure, wound healing trajectories (\% of chronic wound closure vs. time) have been used to describe chronic healing ${ }^{21,22}$. Robson et al. have recently demonstrated the effectiveness of using wound healing trajectories as predictors of therapeutic agents in diabetic foot ulcers, ${ }^{17,23}$ and Hill et al. have reported a similar strategy for pressure ulcers. ${ }^{24}$

Since acute surgical incisions usually heal, 100\% wound closure as recommended by many for chronic wounds is not a reasonable outcome measure for acute wounds. Similarly, survival study methods as described by Kaplan and Meier are not germane since there are not usually a significant number of patients who do not heal. ${ }^{25}$ Finally, analogous to open wounds, the rate of change (increase) of incision tensile strength progressively decreases as time passes and 100\% unwounded tissue strength is never achieved, making the endpoint definition of "healed" vague. Rather, using a dynamic healing trajectory such as gain in wound strength vs. time will allow curves to be made for individuals, for experimental groups, or for controls. Hokanson et al. have described how the time to reach an arbitrary fraction of total healing, in this case the Newtons of breaking strength, can be used. ${ }^{26}$ Distributions of these fractional strength gain times and statistical differences in the distributions can then be determined using statistical methodology similar that used for failure time of survival analysis. $^{27}$

Healing trajectories provide more information about the entire continuum of the wound healing processes. ${ }^{17}$ If total incisional repair failure is the only outcome measure for acute wound healing, then statistical comparison at this extreme may be uninformative and stresses the need for statistical techniques that allow dynamic comparisons at biologically meaningful interim values. ${ }^{26} \mathrm{Po}^{-}$ lansky and Rijswijk have suggested that healing time curves (wound healing trajectories) are a "moving picture" of healing, providing more detail than the "snapshot" approach in which only the proportion of patients healed at the end of a study is assessed. ${ }^{28}$

How can the acute wound healing trajectory be used as an outcome determinant? One approach is to first dissect the traditional acute wound healing curve into its major components; the hemostasis/inflammatory phase, fibroplastic phase, and remodeling phase. We hypothesize that the early lag phase during acute healing is pri- marily the result of the amount of time required to activate the necessary molecular and cellular components of early tissue repair. Next, the potent cytokine and tissue growth factor signaling peptides believed to be most important to each phase is identified. Finally, a technique for delivering the inflammatory and or growth factor mediator to the wound environment sooner or in a more sustained manner is developed and the effect on the acute wound healing trajectory is measured. In animal models, one technique for shifting the inflammatory lag phase of wound healing to the left is to therapeutically activate the target tissue prior to wounding. "Priming" early acute wound healing using classic wound repair cytokines such as platelet-derived growth factor, transforming growth factor- $\beta_{2}$ and interleukin- $1 \beta$ has been shown to preactivate the humoral and cellular elements of acute tissue repair and to accelerate the recovery of tissue breaking strength following incision. ${ }^{29}$ Transforming growth factor- $\beta$ has also been used to successfully shift the proliferative phase of incisional healing to the left. ${ }^{30}$ In these studies, dermal wounds were treated at the time of incision and a left-ward shift of the acute healing curve again was observed. Finally, the remodeling phase of acute tissue repair has also been accelerated using electrical field stimulation across the wound. ${ }^{31,32}$

Ultimately the strategies developed in the laboratory for optimizing acute wound healing trajectories must be translated into meaningful clinical approaches. As stated earlier, there are inherent ethical and practical difficulties in manipulating otherwise normal acute wounds. Incisional wound breaking strength is usually the most clinically relevant outcome measure for acute surgical wounds but direct measurement requires wound biopsies. Although this has been reported in humans, all reports have been of relatively few samples often on volunteers and usually at one point in time making the generation of an acute wound healing trajectory impossi-

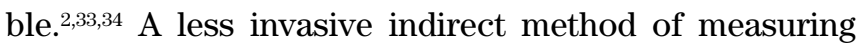
gain in incisional breaking strength in vivo is by measuring nondisruptive acute wound deformation. One device places an acute wound under multiaxial stress using a wound vacuum chamber. Acute wound deformation is then quantified and correlated to gain in wound tensile strength. $^{35,36}$ This nondisruptive methodology might allow for the generation of clinically relevant human acute wound healing trajectories, although limited to the dermis.

An alternative approach for measuring human acute wound healing is through the development and validation of surrogate markers. Acute wound drain fluid, for example, has been biochemically analyzed over time for $\mathrm{pH}$ and hydroxyproline or procollagen fragment levels. ${ }^{37}$ 
These data are, however, variable and difficult to analyze leaving many methodological problems to be resolved. Tissue/wound oxygen partial pressure $\left(\mathrm{P}_{\mathrm{T}} \mathrm{O}_{2}\right)$ is important to many components of acute wound healing but its application is probably limited in most acute wounds. It is fairly well accepted that the biochemical kinetics of collagen deposition and cross-linking are maximized at a $\mathrm{P}_{\mathrm{T}} \mathrm{O}_{2}$ of approximately $25 \mathrm{mmHg}$ and that collagen deposition is independent of absolute $\mathrm{P}_{\mathrm{T}} \mathrm{O}_{2}$ values above this. ${ }^{38}$ Most incisional wounds would be expected to have $\mathrm{P}_{\mathrm{T}} \mathrm{O}_{2}$ values at or above this value fairly early on limiting its usefulness in most normal wounds. Pain scores and quality of life endpoints have also been used to measure the progress of acute wound healing although experience in clinical acute wound healing studies is limited. ${ }^{39}$ Time to suture removal has been correlated with acute wound outcomes in at least one report studying the effect of intraoperative hypothermia on surgical wound infection rates. ${ }^{40}$ It is therefore conceivable that time to suture removal could serve as a useful surrogate marker as an acute wound healing endpoint, but again, the generation of an acute wound healing trajectory is impossible with one time point. Finally, wound infection rate itself may serve as a surrogate marker for acute wound healing. Especially in colorectal surgery where wound infection rates remain 9-27\% it might be theorized that accelerated wound healing would itself diminish wound infection rates through either a decrease in the duration of the wound or through improvement in the quality of the acute wound environment. ${ }^{41}$

Newer approaches to measuring the progress of acute wound healing include laser Doppler imaging. This technique provides a noninvasive method for quantifying wound perfusion and has been particularly useful in the management of burned patients. Many experimental and clinical reports describe the importance of tissue perfusion to tissue repair following injury and its use as a marker of acute wound healing success. ${ }^{42}$ Advances in ultrasound imaging technology may also make it possible to follow the progression of acute wound healing not only in the dermis but in inaccessible tissue planes such as the fascia of the abdominal wall or the GI tract. ${ }^{43}$ Acute scar thickness or wound-edge gapping, for example, could be measured over time. The main problem with each of these technical advances remains correlating the surrogate information back to a human acute wound breaking strength trajectory for validation.

It is clear from preclinical studies that the acute wound healing trajectory can be shifted to the left from a "normal" curve to an accelerated or more "ideal" curve. If a clinical trial were to be conducted using cytokines such as used in the earlier examples this shift of trajec- tory could be used as an outcome determinant. Hopefully, all incisions in such a trial would heal. It would be the acceleration of time to gain of wound strength or a surrogate marker of wound strength that would be important. For each group the time required to gain various strengths could be determined and the time to reach $75 \%$ or $85 \%$ of normal strength could be evaluated. These distributions of times to an event (e.g., 85\% normal strength) could then undergo standard statistical failure time analysis. ${ }^{27}$ These techniques could then be used to compare two experimental groups or generalized for comparing multiple groups. It will be through novel approaches such as these that the basic science of acute wound healing is inevitably applied to the problem of acute surgical wounds.

\section{ACKNOWLEDGMENTS}

Preparation of this article was supported in part by a Veterans Administration Merit Review Grant.

\section{REFERENCES}

1. National Center for Health Statistics. Detailed diagnoses and procedures. National hospital discharge survey. Series 13 No. 122, 1995.

2. Carlson MA. Acute wound failure. Wound healing. Surg Clin N Am 1997;77:607-35.

3. Cooper DM. Clinical assessment/measurement of healing: evolution and status. Clin Mat 1991;8:263-71.

4. Mani RJ. Science of measurements in wound healing. Wound Rep Reg 1999;7:330-4.

5. Dent TL. Training, credentialing and evaluation in laparoscopic surgery. Surg Clin N Am 1992;72:1003-111.

6. Viidik A, Gottrup F. Mechanics of healing soft tissue wounds. In: Schmid-Schonbein GW, Woo SL-Y, Zweifach BW, editors. Frontiers in Biomechanics. New York: Springer, 1986:263-79.

7. Witte MB, Barbul A. General principles of wound healing. Surg Clin N Am 1997;77:509-29.

8. Clark RAF. Wound repair: overview and general considerations. In: Clark RAF, editor. The molecular and cellular biology of wound repair. New York: Plenum Press, 1996:3-35.

9. Iocono JA, Ehrlich HP, Gottrup F, Leaper DJ. The biology of healing. In: Leaper DJ, Harding KG, editors. Wound biology and management. Oxford: Oxford University Press, 1998:10-4.

10. McCarthy K, Henson PM. Induction of lysozomal enzyme secretion by macrophages in response to the purified complement fragments C5a and C5a des-arg. J Immunol 1979;123:2511-7.

11. Pierce GF, Mustoe TA, Altrock BW, Deuel TF, Thomason A. Role of platelet derived growth factor in wound healing. J Cell Biochem 1991;45:319-26.

12. Mulder GD, Brazinsky BA, Harding KG, Agren MS. Factors influencing wound healing. In: Leaper DJ, Harding KG, editors. Wound biology and management. Oxford: Oxford University Press, 1998: 52-69.

13. Riches DWH. Macrophage involvement in wound repair, remodelling and fibrosis. In: Clark, RAF. editor. The molecular and cellular biology of wound repair. New York, Plenum Press, 1996:100-17.

14. Choi M, Rabb H, Arnout MA, Ehrlich HP. Preventing the infiltration of leukocytes by monoclonal antibody prevents the development 
of progressive ischemia in rat burns. Plast Reconst Surg 1995;96: $1177-85$.

15. Richey KJ, Engrav LH, Parlin EG, Murray MJ, Gottlieb JR, Walkinshaw MD. Topical growth factors and wound contraction in the rat. Part I. Literature review and definition of the rat model. Ann Plast Surg 1989;23:159-65.

16. Lazarus GS, Cooper DM, Knighton DR, Margolis DJ, Pecararo DJ, Rodeheaver G. Definitions and guidelines for assessment of wounds and evaluation of healing. Arch Dermatol 1994;130: 489-93.

17. Robson MC, Hill DP, Woodske ME, Steed DL. Wound healing trajectories as predictors of effectiveness of therapeutic agents. Arch Surg 2000;135:773-77.

18. Wright TE, Hill DP, Polo M. The modulation of acute incisional wound healing with $\mathrm{r}-\mathrm{TGF}-\beta_{2}$ and fibrin sealant. Wound Rep Reg 1997;5:A128.

19. Sedgwick CE, Sullivan JT Jr. Abdominal wound disruptions: analysis of 217 cases. Surg Clin N Am 1957;37:731-41.

20. Phillips JD, Kim CS, Fonkalsrud EW, Zeng H, Dindar H. Effects of chronic corticosteroids and vitamin A on the healing of intestinal anastamoses. Am J Surg 1992;163:71-7.

21. Pecoraro RE, Ahroni JH, Boyko EJ, Stensel VL. Chronology and determinants of tissue repair in diabetic lower-extremity ulcers. Diabetes 1991;40:305-13.

22. Tallman P, Muscare E, Carson P, Eaglstein WH, Falanga V. Initial rate of healing predicts complete healing of venous ulcers. Arch Dermatol 1997;133:1231-4.

23. Robson MC, Phillips LG, Thomason A, Robson LE, Pierce GF. Platelet derived growth factor-BB for the treatment of chronic pressure ulcers. Lancet 1992;339:23-5.

24. Hill DP, Payne WG, Robson MC. Wound healing trajectories to determine pressure ulcer treatment efficacy. Wound Rep Regen 1999; 7:A256.

25. Kaplan E, Meier P. Nonparametric estimation from incomplete observation. J Am Stat Assoc 1958;53:457-81.

26. Hokanson JA, Hayward PG, Carney DH, Phillips LG, Robson MC. A mathematical model for the analysis of experimental wound healing data. Wounds 1991;3:213-20.

27. Peto R, Pike G, Armitage P, Breslow E, Cox DR, Howard SV, Mantel M, McPherson K, Smith PG. Design and analysis of randomized clinical trials requiring prolonged observation of each patient. Part II Anal Examples Br J Cancer 1977;35:1-9.

28. Polansky M, van Rijswijk L. Utilizing survival techniques in chronic wound healing studies. Wounds 1994;6:150-8.
29. Smith PD, Kuhn MA, Franz MG, Wachtel TL, Wright TE, Robson MC. Initiating the inflammatory phase of incisional healing prior to tissue injury. J Surg Res 2000;92;11-7.

30. Mustoe TA, Pierce GF, Thomason A, Gramates P, Sporn MB, Deuel TF. Accelerated healing of incisional wounds in rats induced by transforming growth factor-beta. Science 1987;237:1333-6.

31. Robinson KR. The responses of cells to electric fields: a review. J Cell Biol 1985;101:2023-7.

32. Kambic HE, Reyes E, Manning T, Waters KC, Reger SI. Influence of $\mathrm{AC}$ and $\mathrm{DC}$ electrical stimulation on wound healing in pigs: a biomechanical analysis. J Invest Surg 1993;6:535-43.

33. Lindstedt E, Sandblom P. Wound healing in man: tensile strength of healing wounds in some patient groups. Ann Surg 1975;181: $842-6$.

34. Jorgensen LN, Kallehave F, Gottrup F. Are females better healers? A human study. Wound Rep Reg 1997;5:A278.

35. Gingrass M, Perry L, Hill D, Wright T, Robson M, Fisher J. Nondisruptive, in vivo method for biomechanical characterization of linear incision wound healing: preliminary report. Plast Recon Surg 1998;102:801-6.

36. Charles D, Williams K 3rd, Perry LC, Fisher J, Rees R. An improved method of wound disruption and measurement. J Surg Res 1992; $52: 214-8$.

37. Haukipuro K. Synthesis of collagen types I and III in reincised wounds in humans. Br J Surg 1991;78:708-12.

38. Prockop DJ, Kivirikko KI, Tuderman L, Guzman NA. The biosynthesis of collagen and its disorders. N Engl J Med 1979;301:13-23.

39. Akca O, Melischek M, Scheck T, Hellwagner K, Arkilic CF, Kurz A, Kapral S, Heinz T, Lackner FX, Sessler DI. Postoperative pain and subcutaneous oxygen tension. Lancet 1999;354:41-2.

40. Kurz A, Sessler DI, Lenhardt R. Peri-operative normothermia to reduce the incidence of surgical wound infection and shorten hospitalization. N Engl J Med 1996;334:1209-15.

41. Haley RW, Culver DH, Morgan WM, White JW, Emori TG, Hooton TM. Identifying patients at high risk of surgical wound infection: a simple multivariate index of patient susceptibility and wound contamination. Am J Epidemiol 1985;121:206-15.

42. He C, Cherry GW. Measurement of blood flow in patients with leg ulcers. In: Mani R, Falanga V, Shearman CP, Sandeman D, editors. Chronic wound healing; clinical measurements and basic science. London: Harcourt Brace, 1999: 50-67.

43. Gniadecka M, Quirstoff B. Assessment of dermal water by high frequency ultrasound: comparative studies with nuclear magnetic resonance. Br J Dermatol 1993;101:64-8. 